\title{
Inferences for Exponentiated Gamma Constant-Stress Partially Accelerated Life Test Model Based on Generalized Type-I Hybrid Censored Data
}

\author{
Abdalla Rabie, ${ }^{1}$ Abd-EL-Baset A. Ahmad, ${ }^{2}$ Thierno Souleymane Barry (D), ${ }^{3}$ \\ Hassan M. Aljohani $\left(\mathbb{D},{ }^{4}\right.$ Nada M. Alfaer $\left(\mathbb{D},{ }^{4}\right.$ and Abdulaziz S. Alghamdi ${ }^{5}{ }^{5}$ \\ ${ }^{1}$ Department of Mathematics, Faculty of Science, Al-Azhar University, Assiut 71524, Egypt \\ ${ }^{2}$ Department of Mathematics, Faculty of Science, Assiut University, Assiut, Egypt \\ ${ }^{3}$ Mathematics (Statistics Option) Program, Pan African University, Institute of Basic Sciences, \\ Technology and Innovation (PAUISTI), P. O. BOX 62000-00200, Nairobi, Kenya \\ ${ }^{4}$ Department of Mathematics \& Statistics, College of Science, Taif University, P. O. Box 11099, Taif 21944, Saudi Arabia \\ ${ }^{5}$ Department of Mathematics, College of Science \& Arts, King Abdulaziz University, P. O. Box 344, Rabigh 21911, Saudi Arabia
}

Correspondence should be addressed to Thierno Souleymane Barry; thsoulb15@gmail.com

Received 16 September 2021; Accepted 29 November 2021; Published 27 December 2021

Academic Editor: Alexander Hošovský

Copyright $\odot 2021$ Abdalla Rabie et al. This is an open access article distributed under the Creative Commons Attribution License, which permits unrestricted use, distribution, and reproduction in any medium, provided the original work is properly cited.

In this paper, the exponentiated gamma distribution (EGD) with generalized Type-I hybrid censored data under constant-stress partially accelerated life test (CSPALT) model is considered. The Bayesian and E-Bayesian estimation methods, as well as the maximum likelihood estimation method, are discussed for the parameter of the distribution and the acceleration factor. The E-Bayesian and Bayesian estimates are derived by using the squared error loss (SEL) and the LINEX loss functions. The MCMC method is applied for deriving the Bayesian and then E-Bayesian estimates. Moreover, a real data set is given for the illustrative purpose. After all, an evaluation is performed for the results of the proposed methods.

\section{Introduction}

Many types of censoring schemes are used in the last decades. Type-I and Type-II censoring methods are still the most often used censoring schemes. In Type-I censoring, the test is terminated at a specified time $\tau$ and the number of failures is random. In Type-II censoring, the test is ended after obtaining a prefixed number of failures while the time of the test is random. In these two types, the experimenter does not know when he will finish the test (as in Type-II) or he can get the required number before time $\tau$ (as in Type-I). To overcome these disadvantages of Type-I and Type-II, Epstein [1] introduced a mixture of Type-I and Type-II and referred to as hybrid censoring scheme (HCS) mainly, TypeI HCS and Type-II HCS. In Type-I HCS, the test is terminated at a random time $T_{*}=\min \left\{X_{r: n}, T\right\}$, where $r \in\{1,2, \ldots, n\}$ and $T \in(0, \infty)$ are fixed from the begining of the test. Regarding Type-II HCS, the examination is terminated at a random point of time, and let us say it $T^{*}=\max \left\{X_{r: n}, T\right\}$. Also, these schemes have drawbacks like having a few number of failures or not knowing the maximum time to finish the test. Therefore, Chandrasekar et al. [2] proposed an efficient and new censoring scheme which known as the generalized hybrid censoring schemes (generalized HCSs) to overcome drawbacks of HCSs. These schemes are considered as an extension of Type-I and TypeII HCSs. Therefore, we can notice that two types of censoring schemes are defined along these lines.

Generalized Type-I HCS: let $k, r \in\{1,2, \ldots, n\}$ be integers and $k<r<n$, with time point $T \in(0, \infty)$. In this scheme, the test is ended at $\min \left\{X_{r: n}, T\right\}$ when the $k$-th failure happens before $T$. If the $k$-th failure is occurred next to $T$, the experiment is terminated at $X_{k: n}$. Generalized TypeII HCS: set $r \in\{1,2, \ldots, n\}$ with time points $T_{1}, T_{2} \in(0, \infty)$ 
where $T_{1}<T_{2}$. If the $r$-th failure occurs sooner than time $T_{1}$, the experiment is finished at $T_{1}$. If the $r$-th failure is obtained between $T_{1}$ and $T_{2}$, the experiment is terminated at $X_{r: n}$. In the end, if the $r$-th failure occurs after time $T_{2}$, the test is terminated at $T_{2}$. These schemes are studied by many authors such as Huang and Yang [3]; Rabie, and Li [4]; and Rabie and Li [5].

Due to the continuous enhancement in industrial design, it is difficult to get enough information of the products in the reliability tests under normal use conditions. Therefore, the accelerated life test (ALT) and partially accelerated life test (PALT) are used for this purpose. In these tests, a sample is subjected to more severe operating conditions than normal use conditions to obtain rapid failures. ALT items are allocated only in accelerated condition, while PALT is applied with normal and accelerated use conditions. This technique of tests results in shorter lifetimes than under normal use conditions. Extreme stress can be applied in several ways; the most common methods are step-stress and constant-stress as designated in Nelson [6]. In step-stress PALT, units first run at normal use condition; if units do not fail at a specified time, they allocated at accelerated use condition. Under constant stress, test items are divided into two groups: one of them runs at normal use conditions and the other is subjected to accelerated use conditions. We focus on the CSPALT model with generalized Type-I HCS in this paper.

1.1. The Model Description and Test Procedures. The exponentiated gamma distribution (EGD) was suggested by Gupta et al. [7] as an alternative to Weibull and gamma distributions. This study suggested that the EGD can present a better fit to the real data set than the GD. The cumulative distribution function (CDF), probability density function $(\mathrm{PDF})$, and the reliability function $(\mathrm{R}(\mathrm{t}))$ of the EGD are written, respectively, in the forms as follows:

$$
\begin{aligned}
& F(x ; \theta)=\left(1-e^{-x}(x+1)\right)^{\theta}, \quad x>0,(\theta>0), \\
& f(x ; \theta)=\theta x e^{-x}\left(1-e^{-x}(x+1)\right)^{\theta-1}, \quad x>0,(\theta>0), \\
& R(x ; \theta)=1-\left(1-e^{-x}(x+1)\right)^{\theta}, \quad x>0,(\theta>0),
\end{aligned}
$$

where $\theta$ is the shape parameter. It is noted that when $\theta=1$, the EGD turns into $G(2,1)$; for more details, one can refer to Shawky and Bakoban [8-10], Singh et al. [11]; Khan and Kumar [12]; Ghanizadeh et al. [13]; and Feroze and Aslam [14].

Figure 1 shows the plots of the shape of the PDF and CDF of the EGD distribution; it can be seen that the PDF has a unique mode as the parameter $\theta$ decreases. The distance between the shape of $\mathrm{CDF}$ increases as the parameter $\theta$ increases.

In CSPALT, a sample of size $n$ of test items is divided into two groups $n_{1}$ and $n_{2}$ chosen randomly among $n$ items. $n_{1}$ items run at normal use conditions, while $n_{2}$ items are allocated at accelerated use conditions at the same time. The experiment is planned to continue at most until time $T$ in both normal use and accelerated use conditions. We desire to obtain $r_{1}$ and $r_{2}$ failures out of $n_{1}$ and $n_{2}$, respectively. And a bare minimum acceptable number of failures are $k_{1}$ and $k_{2}$ from $n_{1}$ and $n_{2}$, respectively. According to the CSPALT model under generalized Type-I HCS, one can note the following three cases of censored data. For normal use conditions, we observe

$$
\begin{aligned}
& \text { Case I: }\left\{X_{1: n_{1}}<X_{2: n_{1}}<\cdots<X_{k_{1}: n_{1}}\right\} \text { if } X_{k_{1}: n_{1}}>T \\
& \text { Case II: }\left\{X_{1: n_{1}}<\cdots<X_{k_{1}: n_{1}}<\cdots<X_{r_{1}: n_{1}}\right\} \text { if } X_{r_{1}: n_{1}}<T \\
& \text { Case III: }\left\{X_{1: n_{1}}<\cdots<X_{k_{1}: n_{1}}<\cdots<X_{d_{1}: n_{1}}\right\} \text { if } \\
& X_{r_{1}: n_{1}}>T
\end{aligned}
$$

where $d_{1}$ denotes the number of failures occurring up to time $T$ in the case of normal use conditions.

Also, for accelerated use conditions, we observe the following three cases:

$$
\begin{aligned}
& \text { Case 1: }\left\{Y_{1: n_{2}}<Y_{2: n_{2}}<\cdots<Y_{k_{1}: n_{2}}\right\} \text { if } Y_{k_{2}: n_{2}}>T \\
& \text { Case 2: }\left\{Y_{1: n_{2}}<\cdots<Y_{k_{2}: n_{2}}<\cdots<Y_{r_{2}: n_{2}}\right\} \text { if } Y_{r_{2}: n_{2}}<T \\
& \text { Case 3: }\left\{Y_{1: n_{2}}<\cdots<Y_{k_{2}: n_{2}}<\cdots<Y_{d_{2}: n_{2}}\right\} \text { if } Y_{r_{2}: n_{2}}>T
\end{aligned}
$$

where $Y=\lambda^{-1} X, \lambda$ is the acceleration factor, and $d_{2}$ stands for the number of failures occurring up to $T$ in the case of accelerated use conditions.

The remainder of this article is organised as follows: In Section 2, the maximum likelihood based on CSPALT generalized Type-I HCS is discussed. In Section 3, the Bayesian estimation is studied under the SEL and LINEX loss functions by using the MCMC method. In Section 4, we present the Bayesian estimates based on the MCMC method. In Section 5, we present the E-Bayesian estimates based on the SEL and LINEX loss functions. In Section 6, we present the simulation study of the algorithm. We examine the flexibility of the distribution to fit the accelerated data in Section 7 , so we provided a real data example, and the numerical results concluded are presented to asses the performance of the distribution.

\section{The Likelihood Function Based on Constant- Stress Generalized Type-I Hybrid Censoring Scheme}

We assume that $X_{1}, X_{2}, \ldots, X_{n_{1}}$ are $n_{1}$ observations of failure lifetimes under typical usage conditions that follows the generalized Type-I HCS, and $Y_{1}, Y_{2}, \ldots, Y_{n_{2}}$ are $n_{2}$ observations of breakdown lifetimes under accelerated usage conditions that follows the generalized Type-I HCS. The lifespan of test items is determined by EGD. So we can refer to the PDF under typical usage situation as in equation (2), and the following PDF is presented for a product in an accelerated consumption stage which can be written as the following:

$$
\begin{array}{r}
f(y ; \theta, \lambda)=\theta \lambda^{2} y e^{-\lambda y}\left(1-e^{-\lambda y}(\lambda y+1)\right)^{\theta-1}, \\
y, \theta>0, \lambda>1,
\end{array}
$$

where $Y=\lambda^{-1} X$. Therefore, if $d_{1}$ denotes failures number obtained before $T$, so we can write the likelihood equation under generalized Type-I HCS for $\left(x_{j} ; \theta\right), j=1,2, \ldots, n_{1}$, 


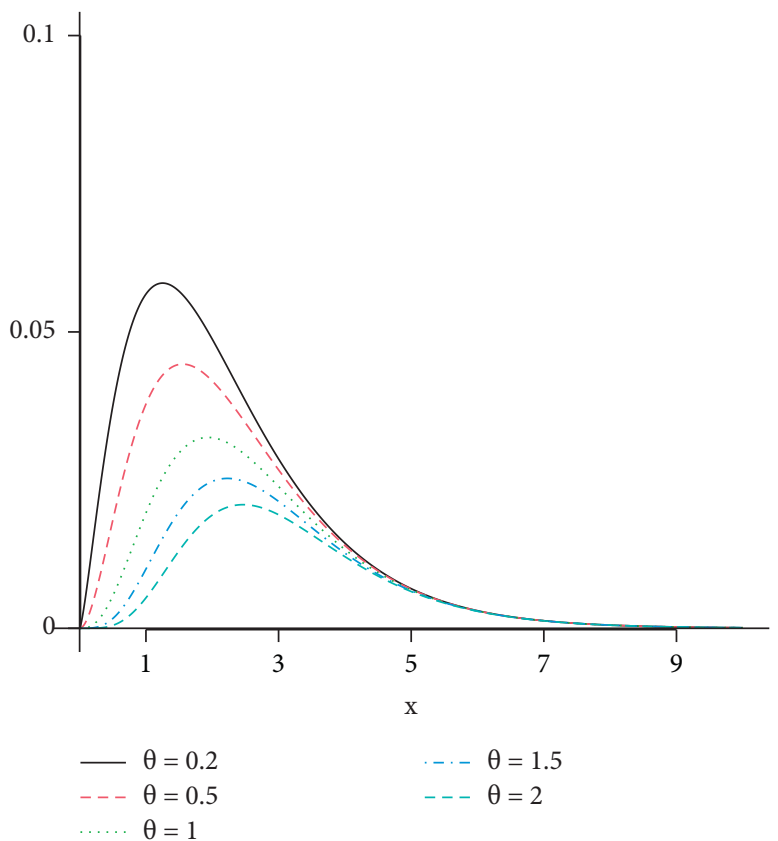

(a)

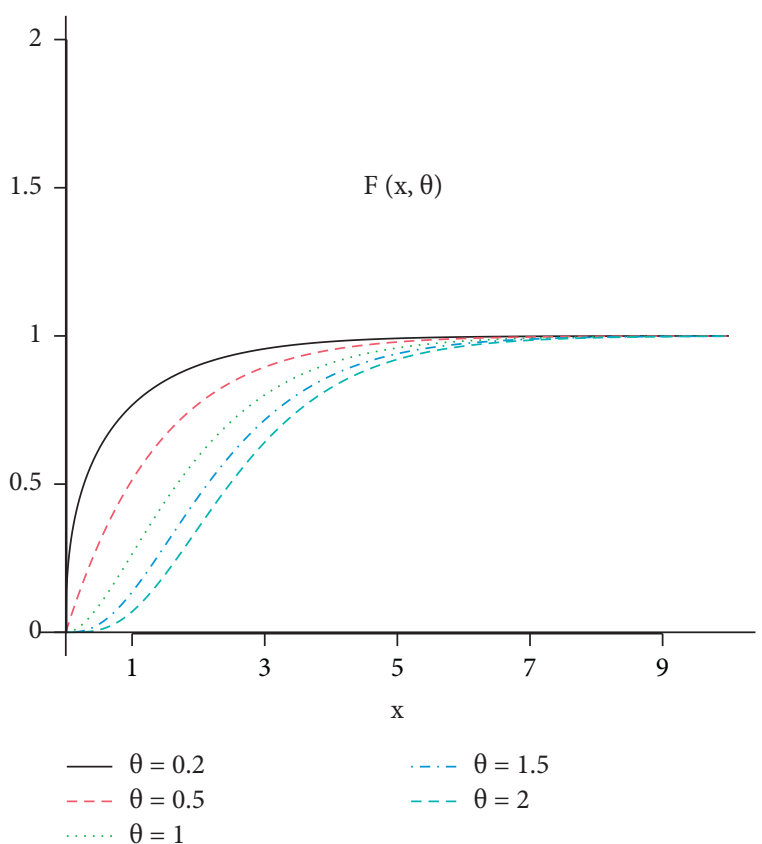

(b)

Figure 1: Plots the shapes of the PDF (a) and CDF (b) of the EGD.

without the multiplicative constant in usual usage is provided as

$$
L(\theta)=\left\{\begin{array}{l}
\theta^{k_{1}}\left\{1-\varphi_{k_{1}}^{\theta}\right\}^{n_{1}-k_{1}} \prod_{i=1}^{k_{1}} x_{i} e^{-x_{i}} \varphi_{i}^{\theta-1}, \\
d_{1}=0,1, \ldots, k_{1}-1, \\
\theta^{d_{1}}\left\{1-\varphi_{T}^{\theta}\right\}^{n_{1}-d_{1}} \prod_{i=1}^{d_{1}} x_{i} e^{-x_{i}} \varphi_{i}^{\theta-1} \\
d_{1}=k_{1}, k_{1}+1, \ldots, r_{1}-1, \\
\theta^{r_{1}}\left\{1-\varphi_{r_{1}}^{\theta}\right\}^{n_{1}-r_{1}} \prod_{i=1}^{r_{1}} x_{i} e^{-x_{i}} \varphi_{i}^{\theta-1} \\
d_{1}=r_{1},
\end{array}\right.
$$

where $\varphi_{t}=1-e^{-t}(t+1)$, and for $\left(y_{j} ; \theta, \lambda\right) ; j=1,2, \ldots, n_{2}$, if $d_{2}$ indicates the number of failures that occur prior to the specified period $T$, we can refer to the likelihood equations without the multiplicative constant in accelerated usage which is provided as

$$
L(\theta, \lambda)=\left\{\begin{array}{l}
\theta^{k_{2}} \lambda^{2 k_{2}}\left\{1-\psi_{k_{2}}^{\theta}\right\}^{n_{2}-k_{2}} \prod_{j=1}^{k_{2}} y_{j} e^{-\lambda y_{j}} \psi_{j}^{\theta-1}, \\
d_{2}=0,1, \ldots, k_{2}-1, \\
\theta^{d_{2}} \lambda^{2 d_{2}}\left\{1-\psi_{T}^{\theta}\right\}^{n_{2}-d_{2}} \prod_{j=1}^{d_{2}} y_{j} e^{-\lambda y_{j}} \psi_{j}^{\theta-1}, \\
d_{2}=k_{2}, k_{2}+1, \ldots, r_{2}-1, \\
\theta^{r_{2}} \lambda^{2 r_{2}}\left\{1-\psi_{r_{2}}^{\theta}\right\}^{n_{2}-r_{2}} \prod_{j=1}^{r_{2}} y_{j} e^{-\lambda y_{j}} \psi_{j}^{\theta-1}, \\
d_{2}=r_{2},
\end{array}\right.
$$

where $\psi_{t}=1-e^{-\lambda t}(\lambda t+1)$. By combining equations (5) and (6), the total likelihood function for $\left\{\left(x_{i} ; \theta\right),\left(y_{j} ; \theta, \lambda\right): i=1\right.$, $\left.\ldots, n_{1} ; j=1, \ldots, n_{2}\right\}$ can be written as follows: 


$$
L(\theta, \lambda)=\left\{\begin{array}{l}
\theta^{k_{1}+k_{2}} \lambda^{2 k_{2}}\left\{1-\varphi_{k_{1}}^{\theta}\right\}^{n_{1}-k_{1}}\left\{1-\psi_{k_{2}}^{\theta}\right\}^{n_{2}-k_{2}} \prod_{i=1}^{k_{1}} x_{i} e^{-x_{i}} \varphi_{i}^{\theta-1} \\
\times \prod_{j=1}^{k_{2}} y_{j} e^{-\lambda y_{j}} \psi_{j}^{\theta-1}, d_{1}=0,1, \ldots, k_{1}-1, d_{2}=0,1, \ldots, k_{2}-1, \\
\theta^{d_{1}+d_{2}} \lambda^{2 d_{2}}\left\{1-\varphi_{T}^{\theta}\right\}^{n_{1}-d_{1}}\left\{1-\psi_{T}^{\theta}\right\}^{n_{2}-d_{2}} \prod_{i=1}^{d_{1}} x_{i} e^{-x_{i}} \varphi_{i}^{\theta-1} \\
\times \prod_{j=1}^{d_{2}} y_{j} e^{-\lambda y_{j}} \psi_{j}^{\theta-1}, d_{1}=k_{1}, k_{1}+1, \ldots, r_{1}-1, d_{2}=k_{2}, k_{2}+1, \ldots, r_{2}-1, \\
\theta^{r_{1}+r_{2}} \lambda^{2 r_{2}}\left\{1-\varphi_{r_{1}}^{\theta}\right\}^{n_{1}-r_{1}}\left\{1-\psi_{r_{2}}^{\theta}\right\}^{n_{2}-r_{2}} \prod_{i=1}^{r_{1}} x_{i} e^{-x_{i}} \varphi_{i}^{\theta-1} \\
\times \prod_{j=1}^{r_{2}} y_{j} e^{-\lambda y_{j}} \psi_{j}^{\theta-1}, d_{1}=r_{1}, d_{2}=r_{2} .
\end{array}\right.
$$

2.1. Maximum Likelihood Estimation Method. As it is stated, the log-likelihood is monotonically increasing so maximizing the likelihood function is equivalent to maximizing the log-likelihood. By calculating the log of the expression (7), as proceeds, we obtain the log-likelihood function:

$$
\begin{aligned}
& \ell=\ln L(\theta, \lambda) \\
& =\left\{\begin{array}{l}
\left(k_{1}+k_{2}\right) \ln \theta+2 k_{2} \ln \lambda+\left(n_{1}-k_{1}\right) \ln \left\{1-\varphi_{k_{1}}^{\theta}\right\}+\left(n_{2}-k_{2}\right) \ln \left\{1-\psi_{k_{2}}^{\theta}\right\} \\
+\sum_{i=1}^{k_{1}} \ln x_{i}-\sum_{i=1}^{k_{1}} x_{i}+(\theta-1) \sum_{i=1}^{k_{1}} \ln \left(\varphi_{i}\right)+\sum_{j=1}^{k_{2}} \ln y_{j}-\sum_{j=1}^{k_{2}} \lambda y_{j}+(\theta-1) \sum_{j=1}^{k_{2}} \ln \left(\psi_{j}\right), \\
d_{1}=0,1, \ldots, k_{1}-1, d_{2}=0,1, \ldots, k_{2}-1, \\
\left(d_{1}+d_{2}\right) \ln \theta+2 d_{2} \ln \lambda+\left(n_{1}-d_{1}\right) \ln \left\{1-\varphi_{T}^{\theta}\right\}+\left(n_{2}-d_{2}\right) \ln \left\{1-\psi_{T}^{\theta}\right\} \\
+\sum_{i=1}^{d_{1}} \ln x_{i}-\sum_{i=1}^{d_{1}} x_{i}+(\theta-1) \sum_{i=1}^{d_{1}} \ln \left(\varphi_{i}\right)+\sum_{j=1}^{d_{2}} \ln y_{j}-\lambda \sum_{j=1}^{d_{2}} y_{j}+(\theta-1) \sum_{j=1}^{d_{2}} \ln \left(\psi_{j}\right), \\
d_{1}=k_{1}, k_{1}+1, \ldots, r_{1}-1, d_{2}=k_{2}, k_{2}+1, \ldots, r_{2}-1, \\
\left(r_{1}+r_{2}\right) \ln \theta+2 r_{2} \ln \lambda+\left(n_{1}-r_{1}\right) \ln \left\{1-\varphi_{r_{1}}^{\theta}\right\}+\left(n_{2}-r_{2}\right) \ln \left\{1-\psi_{r_{2}}^{\theta}\right\} \\
+\sum_{i=1}^{r_{1}} \ln x_{i}-\sum_{i=1}^{r_{1}} x_{i}+(\theta-1) \sum_{i=1}^{r_{1}} \ln \left(\varphi_{i}\right)+\sum_{j=1}^{r_{2}} \ln y_{j}-\lambda \sum_{j=1}^{r_{2}} y_{j}+(\theta-1) \sum_{j=1}^{r_{2}} \ln \left(\psi_{j}\right), \\
d_{1}=r_{1}, d_{2}=r_{2} .
\end{array}\right.
\end{aligned}
$$

Set the first derivatives of equation (8) regarding $\theta$ and $\lambda$ to zero and solve the following equations numerically:

$$
\begin{aligned}
& \frac{\partial \ell}{\partial \theta}=0, \\
& \frac{\partial \ell}{\partial \lambda}=0,
\end{aligned}
$$

to get maximum likelihood estimates (MLEs) of $\theta$ and $\lambda$.

\section{Bayesian Analysis}

In this part of the paper, we made the most important estimation technique which is the Bayesian estimation for the two parametres $\theta$ and $\lambda$. We made estimation using diffirent estimation loss functions such as the SEL and LINEX loss 
functions. We assumed that the test follows CSPALT generalized Type-I hybrid censored sample from EGD. We uesd gamma prior for the two parameters $\theta$ and $\lambda$ like gamma $\left(a_{1}, a_{2}\right)$ and gamma $\left(b_{1}, b_{2}\right)$, respectively. So, we can write the joint prior PDF equation of the two parameters of the distribution $\theta$ and $\lambda$, and it will have the following form:

$$
\begin{array}{r}
\pi(\theta, \lambda)=\pi_{1}(\theta) \pi_{2}(\lambda) \\
\propto \theta^{a_{1}-1} \lambda^{b_{1}-1} e^{-\left(a_{2} \theta+b_{2} \lambda\right),}
\end{array}
$$

where

$$
\begin{gathered}
\pi_{1}(\theta) \propto \theta^{a_{1}-1} \exp \left(-a_{2} \theta\right),\left(a_{1}, a_{2}>0\right), \theta>0, \\
\pi_{2}(\lambda) \propto \lambda^{b_{1}-1} \exp \left(-b_{2} \lambda\right),\left(b_{1}, b_{2}>0\right), \lambda>1 .
\end{gathered}
$$

Then, the joint posterior PDF of $\theta$ and $\lambda$ is written from (7) and (10) as follows:

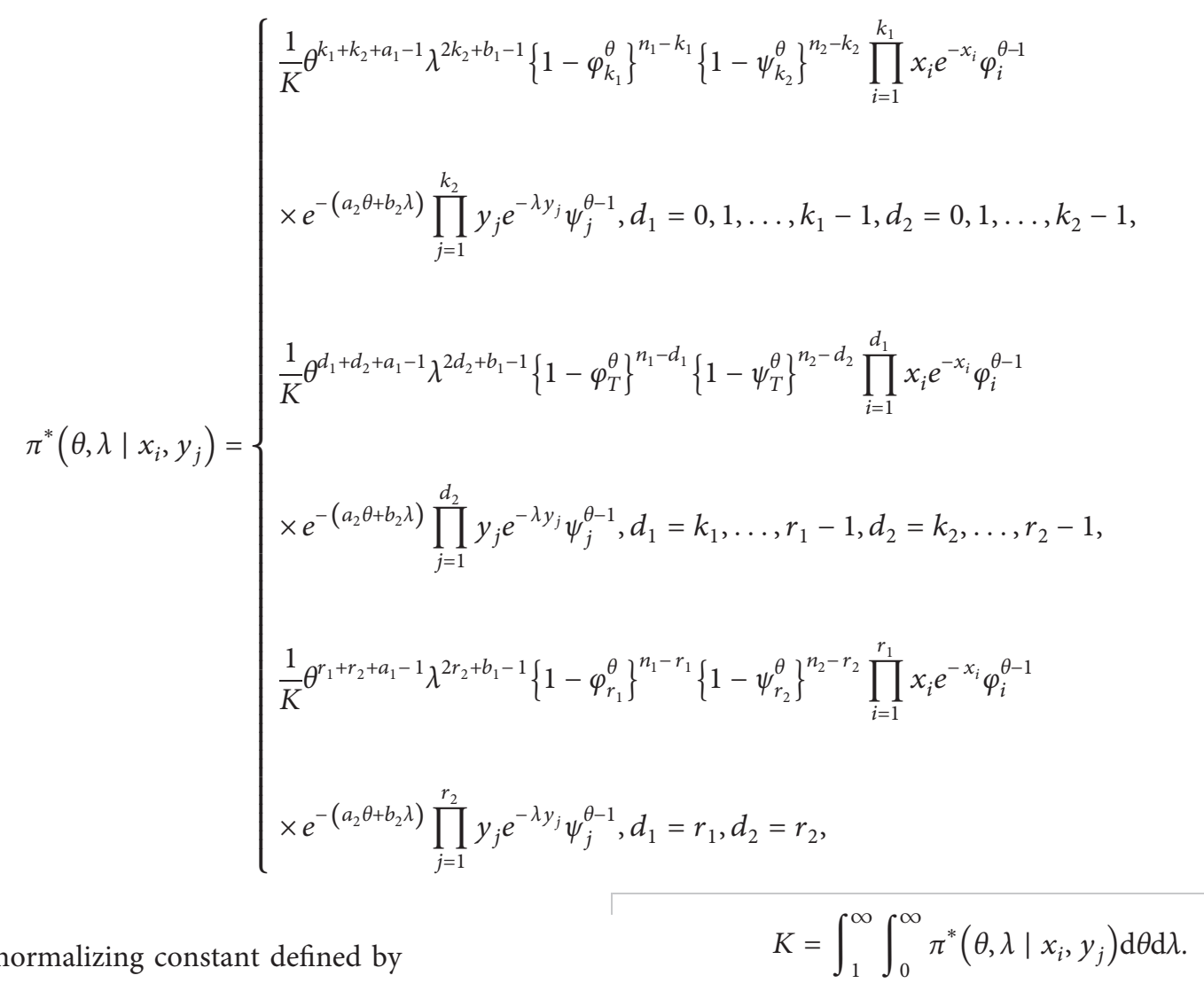

where $K$ is a normalizing constant defined by 
Under the SEL function, the Bayesian estimate of any function $u(\theta, \lambda)$ of $\theta$ and $\lambda$ is given by

$$
\begin{aligned}
& \widehat{u}_{B S}= E[u(\theta, \lambda) \mid x, y] \\
&= \int_{1}^{\infty} \int_{0}^{\infty} u(\theta, \lambda) \pi^{*}\left(\theta, \lambda \mid x_{i}, y_{j}\right) \mathrm{d} \theta \mathrm{d} \lambda \\
& \frac{1}{K} \int_{1}^{\infty} \int_{0}^{\infty} u(\theta, \lambda) \theta^{k_{1}+k_{2}+a_{1}-1} \lambda^{2 k_{2}+b_{1}-1}\left\{1-\varphi_{k_{1}}^{\theta}\right\}^{n_{1}-k_{1}}\left\{1-\psi_{k_{2}}^{\theta}\right\}^{n_{2}-k_{2}} \prod_{i=1}^{k_{1}} x_{i} e^{-x_{i}} \varphi_{i}^{\theta-1} \\
& \times e^{-\left(a_{2} \theta+b_{2} \lambda\right)} \prod_{j=1}^{k_{2}} y_{j} e^{-\lambda y_{j}} \psi_{j}^{\theta-1} \mathrm{~d} \theta \mathrm{d} \lambda, d_{1}=0,1, \ldots, k_{1}-1, d_{2}=0,1, \ldots, k_{2}-1, \\
& \frac{1}{K} \int_{1}^{\infty} \int_{0}^{\infty} u(\theta, \lambda) \theta^{d_{1}+d_{2}+a_{1}-1} \lambda^{2 d_{2}+b_{1}-1}\left\{1-\varphi_{T}^{\theta}\right\}^{n_{1}-d_{1}}\left\{1-\psi_{T}^{\theta}\right\}^{n_{2}-d_{2}} \prod_{i=1}^{d_{1}} x_{i} e^{-x_{i}} \varphi_{i}^{\theta-1} \\
& \times e^{-\left(a_{2} \theta+b_{2} \lambda\right)} \prod_{j=1}^{d_{2}} y_{j} e^{-\lambda y_{j}} \psi_{j}^{\theta-1} \mathrm{~d} \theta \mathrm{d} \lambda, d_{1}=k_{1}, \ldots, r_{1}-1, d_{2}=k_{2}, \ldots, r_{2}-1, \\
& \frac{1}{K} \int_{1}^{\infty} \int_{0}^{\infty} u(\theta, \lambda) \theta^{r_{1}+r_{2}+a_{1}-1} \lambda^{2 r_{2}+b_{1}-1}\left\{1-\varphi_{r_{1}}^{\theta}\right\}^{n_{1}-r_{1}}\left\{1-\psi_{r_{2}}^{\theta}\right\}^{n_{2}-r_{2}} \prod_{i=1}^{r_{1}} x_{i} e^{-x_{i}} \varphi_{i}^{\theta-1} \\
& \times e^{-\left(a_{2} \theta+b_{2} \lambda\right)} \prod_{j=1}^{r_{2}} y_{j} e^{-\lambda y_{j}} \psi_{j}^{\theta-1} \mathrm{~d} \theta \mathrm{d} \lambda, d_{1}=r_{1}, d_{2}=r_{2} .
\end{aligned}
$$

Based on the LINEX loss function, the Bayesian estimate of $u(\theta, \lambda)$ is given by

$$
\begin{aligned}
\widehat{u}_{B L}= & \frac{-1}{h} \ln E\left[e^{(-h u(\theta, \lambda))} \mid x, y\right] \\
= & \frac{-1}{h} \ln \int_{1}^{\infty} \int_{0}^{\infty} e^{(-h u(\theta, \lambda))} \pi^{*}\left(\theta, \lambda \mid x_{i}, y_{j}\right) \mathrm{d} \theta \mathrm{d} \lambda \\
& \left\{\begin{array}{l}
\frac{-1}{K h} \ln \int_{1}^{\infty} \int_{0}^{\infty} \theta^{k_{1}+k_{2}+a_{1}-1} \lambda^{2 k_{2}+b_{1}-1}\left\{1-\varphi_{k_{1}}^{\theta}\right\}^{n_{1}-k_{1}}\left\{1-\psi_{k_{2}}^{\theta}\right\}^{n_{2}-k_{2}} \prod_{i=1}^{k_{1}} x_{i} e^{-x_{i}} \varphi_{i}^{\theta-1} \\
\times e^{-\left(h u(\theta, \lambda)+a_{2} \theta+b_{2} \lambda\right)} \prod_{j=1}^{k_{2}} y_{j} e^{-\lambda y_{j}} \psi_{j}^{\theta-1} \mathrm{~d} \theta \mathrm{d} \lambda, d_{1}=0,1, \ldots, k_{1}-1, d_{2}=0,1, \ldots, k_{2}-1, \\
\frac{-1}{K h} \ln \int_{1}^{\infty} \int_{0}^{\infty} \theta^{d_{1}+d_{2}+a_{1}-1} \lambda^{2 d_{2}+b_{1}-1}\left\{1-\varphi_{T}^{\theta}\right\}^{n_{1}-d_{1}}\left\{1-\psi_{T}^{\theta}\right\}^{n_{2}-d_{2}} \prod_{i=1}^{d_{1}} x_{i} e^{-x_{i}} \varphi_{i}^{\theta-1} \\
\times e^{-\left(h u(\theta, \lambda)+a_{2} \theta+b_{2} \lambda\right)} \prod_{j=1}^{d_{2}} y_{j} e^{-\lambda y_{j}} \psi_{j}^{\theta-1} \mathrm{~d} \theta \mathrm{d} \lambda, d_{1}=k_{1}, \ldots, r_{1}-1, d_{2}=k_{2}, \ldots, r_{2}-1, \\
\times e^{-\left(h u(\theta, \lambda)+a_{2} \theta+b_{2} \lambda\right)} \prod_{j=1}^{r_{2}} y_{j} e^{-\lambda y_{j}} \psi_{j}^{\theta-1} \mathrm{~d} \theta \mathrm{d} \lambda, d_{1}=r_{1}, d_{2}=r_{2} . \\
\frac{-1}{K h} \ln \int_{1}^{\infty} \int_{0}^{\infty} \theta^{r_{1}+r_{2}+a_{1}-1} \lambda^{2 r_{2}+b_{1}-1}\left\{1-\varphi_{r_{1}}^{\theta}\right\}^{n_{1}-r_{1}}\left\{1-\psi_{r_{2}}^{\theta}\right\}^{n_{2}-r_{2}} \prod_{i=1}^{r_{1}} x_{i} e^{-x_{i}} \varphi_{i}^{\theta-1}
\end{array}\right.
\end{aligned}
$$


It is clear from (14) and (15) that Bayesian estimates of $\theta$ and $\lambda$ cannot be directly calculated, so the MCMC method is used for this purpose.

\section{Bayesian Estimates Using MCMC Method}

Here, we present the MCMC technique to compute and find the Bayesian estimates of $\theta$ and $\lambda$. The conditional posterior PDF of the parameter $\theta$ and the acceleration factor $\lambda$ is, respectively, written as follows:

$$
\pi^{*}\left(\theta \mid \lambda ; x_{i}, y_{j}\right)=\left\{\begin{array}{l}
\theta^{k_{1}+k_{2}+a_{1}-1}\left\{1-\varphi_{k_{1}}^{\theta}\right\}^{n_{1}-k_{1}}\left\{1-\psi_{k_{2}}^{\theta}\right\}^{n_{2}-k_{2}} \prod_{i=1}^{k_{1}} \varphi_{i}^{\theta-1} \\
\times e^{-a_{2} \theta} \prod_{j=1}^{k_{2}} \psi_{j}^{\theta-1}, d_{1}=0,1, \ldots, k_{1}-1, d_{2}=0,1, \ldots, k_{2}-1, \\
\theta^{d_{1}+d_{2}+a_{1}-1}\left\{1-\varphi_{T}^{\theta}\right\}^{n_{1}-d_{1}}\left\{1-\psi_{T}^{\theta}\right\}^{n_{2}-d_{2}} \prod_{i=1}^{d_{1}} \varphi_{i}^{\theta-1} \\
\times e^{-a_{2} \theta} \prod_{j=1}^{d_{2}} \psi_{j}^{\theta-1}, d_{1}=k_{1}, \ldots, r_{1}-1, d_{2}=k_{2}, \ldots, r_{2}-1, \\
\theta^{r_{1}+r_{2}+a_{1}-1}\left\{1-\varphi_{r_{1}}^{\theta}\right\}^{n_{1}-r_{1}}\left\{1-\psi_{r_{2}}^{\theta}\right\}^{n_{2}-r_{2}} \prod_{i=1}^{r_{1}} \varphi_{i}^{\theta-1} \\
\times e^{-a_{2} \theta} \prod_{j=1}^{r_{2}} \psi_{j}^{\theta-1}, d_{1}=r_{1}, d_{2}=r_{2},
\end{array}\right.
$$

and

$$
\pi^{*}\left(\lambda \mid \theta ; x_{i}, y_{j}\right)=\left\{\begin{array}{l}
\lambda^{2 k_{2}+b_{1}-1}\left\{1-\psi_{k_{2}}^{\theta}\right\}^{n_{2}-k_{2}} e^{-b_{2} \lambda} \prod_{j=1}^{k_{2}} e^{-\lambda y_{j}} \psi_{j}^{\theta-1}, \\
d_{1}=0,1, \ldots, k_{1}-1, d_{2}=0,1, \ldots, k_{2}-1, \\
\lambda^{2 d_{2}+b_{1}-1}\left\{1-\psi_{T}^{\theta}\right\}^{n_{2}-d_{2}} e^{-b_{2} \lambda} \prod_{j=1}^{d_{2}} e^{-\lambda y_{j}} \psi_{j}^{\theta-1}, \\
d_{1}=k_{1}, \ldots, r_{1}-1, d_{2}=k_{2}, \ldots, r_{2}-1, \\
\lambda^{2 r_{2}+b_{1}-1}\left\{1-\psi_{r_{2}}^{\theta}\right\}^{n_{2}-r_{2}} e^{-\left(a_{2} \theta+b_{2} \lambda\right)} \prod_{j=1}^{r_{2}} e^{-\lambda y_{j}} \psi_{j}^{\theta-1}, \\
d_{1}=r_{1}, d_{2}=r_{2} .
\end{array}\right.
$$

As it is seen from equations (16) and (17), the conditional posterior PDF of $\theta$ and $\lambda$ does not look like any well-known models. Therefore, we use Metropolis-Hastings techinque to produce samples of $\theta$ and $\lambda$ from the conditional posterior PDF using normal proposal distribution. Posterior samples of $\theta$ and $\lambda$ are, respectively, generated from equations (16) and (17) using the Metropolis-Hastings algorithm.

Step 1: First we initiate with starting values of $\theta$ and $\lambda$ and let it be the MLE values $\left(\widehat{\theta}_{M L E}, \widehat{\lambda}_{M L E}\right)$.

Step 2: take $j=1$.
Step 3: from equation (16), generate $\theta^{(j)}$ and produce samples of $\theta^{(*)}$ from a normal distribution as a proposal distribution.

Step 4: Now we will compute the probability of accepting or rejecting the generated sample which called the acceptance probability by using the following equation:

$$
r\left(\theta^{j-1} \mid \theta^{(*)}\right)=\min \left[1, \frac{\pi^{*}\left(\theta^{(*)} \mid \lambda^{(j-1)}\right)}{\pi^{*}\left(\theta^{(j-1)} \mid \lambda^{(j-1)}\right)}\right] .
$$


Step 5: we will produce samples from the uniform distribution ranged from zero to one as the following: $U \sim U(0,1)$.

Step 6: if $U \leq r\left(\theta^{(j-1)} \mid \theta^{(*)}\right)$, we accept the generated value and assign $\theta^{(*)}=\theta^{(j)}$; else, reject the proposal and put $\theta^{(j-1)}=\theta^{(j)}$.

Step 7: by the same way we will produce and generate $\lambda^{(j)}$ by using equation (17) and generate $\lambda^{(*)}$ using the normal distribution, and we consider it as the proposal distribution.

Step 8: Make a repetition for the steps from step 4 to step 6 for the parameter $\lambda$ too.

Step 9: Assign $j=j+1$.

Step 10: steps $3-9$ are repeated for $N$ repetitions.

Step 11: We can compute the Bayesian estimate values of the two parameters $\theta$ and $\lambda$ using SEL function which are, respectively, as below:

$$
\begin{aligned}
& \hat{\theta}_{B S}=\frac{1}{N-M} \sum_{j=M+1}^{N} \theta^{(j)}, \\
& \hat{\lambda}_{B S}=\frac{1}{N-M} \sum_{j=M+1}^{N} \lambda^{(j)},
\end{aligned}
$$

where $M$ is the number of iterations that are not considerd in the calculation, and sometime we call it nburn iterations.

Step 12: We can compute the Bayesian estimates values of the two parameters $\theta$ and $\lambda$ using LINEX loss function which are, respectively, as below:

$$
\begin{aligned}
& \hat{\theta}_{B L}=\frac{-1}{h} \ln \left[\frac{1}{N-M} \sum_{j=M+1}^{N} e^{-h \theta^{(j)}}\right], \\
& \hat{\lambda}_{B L}=\frac{-1}{h} \ln \left[\frac{1}{N-M} \sum_{j=M+1}^{N} e^{-h \lambda^{(j)}}\right] .
\end{aligned}
$$

\section{E-Bayesian Estimation Method}

The expectation of Bayesian estimation is referred to as "EBayesian estimation" and described as follows.

Definition 1. Let $\hat{\theta}(a, b)$ be continuous, then

$$
\begin{aligned}
\widehat{\delta}_{E B} & =E[\widehat{\delta}(a, b)] \\
& =\iint_{Q} \widehat{\delta}(a, b) \pi(a, b) \mathrm{d} a \mathrm{~d} b,
\end{aligned}
$$

is called the expected Bayesian estimation of $\delta$ (briefly E-Bayesian estimation) where $\widehat{\delta}(a, b)$ is the Bayesian estimate of $\delta$ with hyperparameters $a$ and $b, Q$ is the domain of $(a, b)$, and $\pi(a, b)$ is the prior PDF of $a$ and $b$ over $Q$.

From Han [15], the prior parameters $\left(a_{1}, a_{2}\right)$ and $\left(b_{1}, b_{2}\right)$ must be picked in order to ensure that $\pi_{1}(\theta)$ and $\pi_{2}(\lambda)$ are indeed a pair of declining functions of $\theta$ and $\lambda$, respectively. We can get the differentiation of $\pi_{1}(\theta)$ regarding $\theta$ and $\pi_{2}(\lambda)$ with respect to $\lambda$ as the following two equations:

$$
\begin{aligned}
& \frac{d \pi_{1}(\theta)}{\mathrm{d} \theta} \propto \theta^{a_{1}-2} \exp \left(-a_{2} \theta\right)\left\{\left(a_{1}-1\right)-a_{2} \theta\right\}, \\
& \frac{d \pi_{2}(\lambda)}{\mathrm{d} \lambda} \propto \lambda^{b_{1}-2} \exp \left(-b_{2} \lambda\right)\left\{\left(b_{1}-1\right)-b_{2} \lambda\right\} .
\end{aligned}
$$

When $0<a_{1}<1$ and $a_{2}>0,\left(d \pi_{1}(\theta) / \mathrm{d} \theta\right)<0$, and when $0<b_{1}<1$ and $b_{2}>0,\left(d \pi_{2}(\lambda) / \mathrm{d} \lambda\right)<0$. Thus, $\pi_{1}(\theta)$ and $\pi_{2}(\lambda)$ are decreasing functions for $\theta$ and $\lambda$, respectively. We make the assumption that the hyperparameters $a_{j}$ and $b_{j}, j=1,2$, are independent and have the bivariate PDF given by

$$
\begin{aligned}
& \pi\left(a_{1}, a_{2}\right)=\pi_{1}\left(a_{1}\right) \pi_{2}\left(a_{2}\right), \\
& \pi\left(b_{1}, b_{2}\right)=\pi_{1}\left(b_{1}\right) \pi_{2}\left(b_{2}\right) .
\end{aligned}
$$

In order to get the E-Bayesian estimates of the two parameters $\theta$ and $\lambda$, we suggest the prior PDFs of $\left(a_{1}, a_{2}\right)$ and $\left(b_{1}, b_{2}\right)$ to clarify the impact of them on the E-Bayesian estimates of $\theta$ and $\lambda$. The prior PDFs of $\left(a_{1}, a_{2}\right)$ and $\left(b_{1}, b_{2}\right)$ are, respectively, given as follows:

$$
\begin{aligned}
& \pi_{1}\left(a_{1}, a_{2}\right)=\frac{2 a_{1}}{c_{1}}, \quad 0<a_{1}<1,0<a_{2}<c_{1}, \\
& \pi_{2}\left(a_{1}, a_{2}\right)=\frac{2 a_{2}}{c_{1}^{2}}, \quad 0<a_{1}<1,0<a_{2}<c_{1}, \\
& \pi_{3}\left(a_{1}, a_{2}\right)=\frac{3 a_{2}^{2}}{c_{1}^{3}}, \quad 0<a_{1}<1,0<a_{2}<c_{1},
\end{aligned}
$$

and

$$
\begin{aligned}
& \pi_{1}\left(b_{1}, b_{2}\right)=\frac{2 b_{1}}{c_{2}}, \quad 0<b_{1}<1,0<b_{2}<c_{2}, \\
& \pi_{2}\left(b_{1}, b_{2}\right)=\frac{2 b_{2}}{c_{2}^{2}}, \quad 0<b_{1}<1,0<b_{2}<c_{2}, \\
& \pi_{3}\left(b_{1}, b_{2}\right)=\frac{3 b_{2}^{2}}{c_{2}^{3}}, \quad 0<b_{1}<1,0<b_{2}<c_{2} .
\end{aligned}
$$

5.1. E-Bayesian Estimates Based on the Loss Functions. By substituting from (19) and (26) in (23), the E-Bayesian estimate of $\theta$ using the SEL function can be easily evaluated using the following equation:

$$
\widehat{\theta}_{E B}=E\left[\widehat{\theta_{B}}\left(a_{1}, a_{2}\right)\right]=\iint_{Q} \widehat{\theta_{B}}\left(a_{1}, a_{2}\right) \pi_{i}\left(a_{1}, a_{2}\right) \mathrm{d} a_{1} \mathrm{~d} a_{2}, \quad i=1,2,3,
$$

and the E-Bayesian estimate of $\lambda$ based on the SEL function is computed by substituting in (23) from (20) and (27) as follows: 


$$
\begin{array}{r}
\hat{\lambda}_{E B}=E\left[\hat{\lambda}_{B}\left(b_{1}, b_{2}\right)\right]=\iint_{Q} \hat{\lambda}_{B}\left(b_{1}, b_{2}\right) \pi_{i}\left(b_{1}, b_{2}\right) \mathrm{d} b_{1} \mathrm{~d} b_{2}, \\
i=1,2,3,
\end{array}
$$

where $\widehat{\theta}_{B}\left(a_{1}, a_{2}\right)$ and $\hat{\lambda}_{B}\left(b_{1}, b_{2}\right)$ are the estimates of the Bayesian method for $\theta$ and $\lambda$ by applying the SEL function.

Similarly, by substituting from (21) and (26) in (23), we get the E-Bayesian estimate of $\theta$ based on the LINEX loss function as follows:

$$
\widehat{\theta}_{E B}=E\left[\widehat{\theta}_{B L}\left(a_{1}, a_{2}\right)\right]=\iint_{Q} \widehat{\theta}_{B L}\left(a_{1}, a_{2}\right) \pi_{i}\left(a_{1}, a_{2}\right) \mathrm{d} a_{1} \mathrm{~d} a_{2}, \quad i=1,2,3,
$$

and the E-Bayesian estimate of $\lambda$ based on the LINEX loss function is obtained by substituting in (23) from (22) and (27) as follows:

$$
\hat{\lambda}_{E B}=E\left[\widehat{\lambda}_{B L}\left(b_{1}, b_{2}\right)\right]=\iint_{Q} \widehat{\lambda}_{B L}\left(b_{1}, b_{2}\right) \pi_{i}\left(b_{1}, b_{2}\right) \mathrm{d} b_{1} \mathrm{~d} b_{2}, \quad i=1,2,3,
$$

where $\widehat{\theta}_{B L}\left(a_{1}, a_{2}\right)$ and $\hat{\lambda}_{B L}\left(b_{1}, b_{2}\right)$ are the estimates of $\theta$ and $\lambda$ regarding the Bayesian method under the LINEX loss function. For details, one can see, Han [16]; Jaheen and Okasha [17]; Okasha [18]; Rabie and Li [19]; Rabie and Li [20]; and Rabie [21]. Before progressing, we describe procedures of the simulation study used in this paper.

\section{Simulation Study}

Here, we provide the simulation results according to the following steps:

Specify the values of $n, r_{1}, r_{2}, k_{1}, k_{2}, h, a_{1}, a_{2}, b_{1}, b_{2}$, $c_{1}, c_{2}$, and $T$.

First we use the uniform distribution to genrate a random sample $n$ from $U(0,1)$.

We will choose $n_{1}$ items that are selected randomly, from $n$ items, and subject them to the normal usage situations.

Compute $n_{2}=n-n_{1}$. These units, allocations, are subjected to stress conditions.

Indicate the initial values used in generating data for the two paramters $\theta$ and $\lambda$.

Now we will produce a generalized Type-I hybrid censored sample from the EGD CSPALT model using the inverse function method by solving $U=\left(1-e^{-x}(x+1)\right)^{\theta}$ regarding $x$ for a typically use situations and by solving $U=\left(1-e^{-\lambda y}(\lambda y+1)\right)^{\theta}$ with respect to $y$, for stress use situations.

We build a Markov chain containing 11,000 data by using the Metropolis-Hastings algorithm of $\theta$ and $\lambda$, and we will not consider the first 1000 values as they are very affected with the initial values.
$\widehat{\theta}_{B S}$ and $\hat{\lambda}_{B S}$ are the Bayesian estimation of the two parameters using the SEL function which can be easily computed by using equation (19) and equation (20), respectively.

$\widehat{\theta}_{B L}$ and $\widehat{\lambda}_{B L}$ are the E-Bayesian estimation of the two parameters using the LINEX loss function which can be easily computed by using equation (21) and equation (22), respectively.

$\widehat{\theta}_{E B S}$ and $\hat{\lambda}_{E B S}$ are the E-Bayesian estimation of the two parameters using the SEL function which can be easily computed by using equation (28) and equation (29), respectively.

$\widehat{\theta}_{E B L}$ and $\hat{\lambda}_{E B L}$ are the E-Bayesian estimation of the two parameters using the LINEX loss function which can be easily computed by using equation (30) and (31), equation respectively.

We can compute the mean squared error (MSE) or the estmiated values of the two parameters $\theta$ and $\lambda$, which are, respectively, as follows:

$$
\begin{aligned}
& \operatorname{MSE}(\widehat{\theta})=\frac{1}{1000} \sum_{j=1}^{1000}\left(\widehat{\theta}_{j}-\theta\right)^{2}, \\
& \operatorname{MSE}(\widehat{\lambda})=\frac{1}{1000} \sum_{j=1}^{1000}\left(\widehat{\lambda}_{j}-\lambda\right)^{2},
\end{aligned}
$$

where $\hat{\theta}$ is considerd as the estimated values for $\theta$ and $\hat{\lambda}$ is considerd as the estimated values for $\lambda$.

The numerical outcomes are obtained using MATHEMATICA 8 functions such as FindRoot, NMaximize, NIntegrate, and RandomReal and displayed in Tables 1 and 2. Table 1 contains the numerical values of the Bayesian, E-Bayesian estimates, also it contains the MLEs, beside these values there is MSE of the parameter $\theta$, using the LINEX and SEL functions. Table 2 contains the numerical values of the Bayesian, E-Bayesian estimates, also it contains the MLEs, beside these values there is MSE of the parameter $\lambda$ using LINEX and SEL functions. By observing the results in Tables 1 and 2, we may infer that the E-Bayesian estimate outperforms the Bayesian estimator for the SEL and LINEX loss functions for the two parameters $\theta$ and $\lambda$ as we can easily see it having small values for the MSE. Additionally, when the sample size rises, the MSE of Bayesian and E-Bayesian estimators decreases for a sample of size $n$ and the censoring time $T$ get increased.

\section{Example of Real-Life Data}

In this section, an example of real-life data is provided to investigate the performance of the proposed methods in the application. These data were used by Singh et al. [22], representing the average monthly rainfall obtained from the Information System for Management of Water Resources 
TABle 1: Average estimates, MSE of MLEs, Bayesian, and E-Bayesian estimates for $\theta$ under LINEX and SEL at $\theta=0.7, \lambda=1.2, a_{1}=b_{1}=0.7, a_{2}=1.4, b_{2}=1.2, h=1.5$, and $c_{1}=c_{2}=2$.

\begin{tabular}{|c|c|c|c|c|c|c|c|c|c|c|c|}
\hline \multirow{2}{*}{$\begin{array}{l}\left(n_{1}, n_{2}\right) \\
\left(r_{1}, r_{2}\right)\end{array}$} & \multirow{2}{*}{$\begin{array}{c}T \\
\left(k_{1}, k_{2}\right)\end{array}$} & \multirow{2}{*}{ Criteria } & \multirow{2}{*}{$\widehat{\theta}_{M L E}$} & \multicolumn{4}{|c|}{ Squared error loss } & \multicolumn{4}{|c|}{ LINEX loss } \\
\hline & & & & $\widehat{\theta}_{B S}$ & $\widehat{\theta}_{E B S 1}$ & $\widehat{\theta}_{E B S 2}$ & $\widehat{\theta}_{E B S 3}$ & $\widehat{\theta}_{B L}$ & $\widehat{\theta}_{E B L 1}$ & $\widehat{\theta}_{E B L 2}$ & $\widehat{\theta}_{E B L 3}$ \\
\hline$(20,25)$ & 2 & $\mathrm{~N}$ & 0.75 & 1.14354 & 0.672402 & 0.576345 & 0.51871 & 1.12974 & 0.667409 & 0.572641 & 0.515693 \\
\hline \multirow[t]{3}{*}{$(15,20)$} & $(10,15)$ & MSE & 0.042 & 0.2102 & 0.005419 & 0.018712 & 0.035638 & 0.19527 & 0.005092 & 0.019241 & 0.036446 \\
\hline & & & & 1.06093 & 0.623824 & 0.534706 & 0.581199 & 1.05855 & 0.622986 & 0.534088 & 0.574801 \\
\hline & 2.5 & MSE & 0.033419 & 0.13029 & 0.005811 & 0.027328 & 0.017594 & 0.12858 & 0.005939 & 0.027533 & 0.018685 \\
\hline \multirow{4}{*}{$\begin{array}{l}(30,40) \\
(20,30) \\
\end{array}$} & & Mean & 092618 & 1.04555 & 0.614784 & 0.526958 & 0.474262 & 1.04407 & 0.614261 & 0.526572 & 0.473949 \\
\hline & $(15,20)$ & & 35 & & 0.007264 & 0.029 & 0.050 & & 0.007353 & 0.030078 & \\
\hline & t & & 0.818 & & 0.603538 & 0.517318 & & & 0.603384 & 0.517205 & 0.465494 \\
\hline & 2.5 & MSE & 0.01973 & 0.10656 & 0.009306 & 0.033373 & 0.05495 & 0.10627 & 0.009336 & 0.033415 & 0.054993 \\
\hline \multirow{4}{*}{$\begin{array}{l}(40,50) \\
(30,40) \\
\end{array}$} & & Mean & 0.885694 & 1.03589 & 0.6091 & 0.522087 & 0.469878 & 1.03477 & 0.608703 & 0.52 & 0.469 \\
\hline & $(20,30)$ & MSE & 0.060416 & 0.11304 & 0.008338 & 0.031708 & 0.053001 & 0.11226 & 0.008405 & 0.03181 & 0.053109 \\
\hline & & & & & 0.594057 & 0.509192 & & & 0.594031 & 0.509172 & 0.458257 \\
\hline & 2.5 & MSE & 0.060304 & 0.09629 & 0.011225 & 0.036408 & 0.058433 & 0.09624 & 0.01123 & 0.036416 & 0.05844 \\
\hline
\end{tabular}

TABLE 2: Average estimates, MSE of MLEs, Bayesian, and E-Bayesian estimates for $\lambda$ under LINEX and SEL at $\theta=0.7, \lambda=1.2, a_{1}=b_{1}=0.7, a_{2}=1.4, b_{2}=1.2, h=1.5$, and $c_{1}=c_{2}=2$.

\begin{tabular}{|c|c|c|c|c|c|c|c|c|c|c|c|}
\hline \multirow{2}{*}{$\begin{array}{l}\left(n_{1}, n_{2}\right) \\
\left(r_{1}, r_{2}\right)\end{array}$} & \multirow{2}{*}{$\begin{array}{c}T \\
\left(k_{1}, k_{2}\right)\end{array}$} & \multirow{2}{*}{ Criteria } & \multirow{2}{*}{$\hat{\lambda}_{M L E}$} & \multicolumn{4}{|c|}{ Squared error loss } & \multicolumn{4}{|c|}{ LINEX loss } \\
\hline & & & & $\hat{\lambda}_{B S}$ & $\widehat{\lambda}_{E B S 1}$ & $\hat{\lambda}_{E B S 2}$ & $\hat{\lambda}_{E B S 3}$ & $\hat{\lambda}_{B L}$ & $\hat{\lambda}_{E B L 1}$ & $\widehat{\lambda}_{E B L 2}$ & $\hat{\lambda}_{E B L 3}$ \\
\hline$(20,25)$ & 2 & Mean & 1.01188 & 1.55998 & 1.07015 & 1.07015 & 1.12365 & 1.52509 & 1.05351 & 1.05351 & 1.10534 \\
\hline \multirow{3}{*}{$(15,20)$} & 0 & MSE & 0.123564 & 0.18131 & 0.041203 & 0.041203 & 0.032665 & 0.15184 & 0.043967 & 0.043967 & 0.033679 \\
\hline & \multirow{2}{*}{2.5} & & 1.002 & 1.55565 & 1.06717 & 1.06717 & 1.156 & 1.52002 & 1.0502 & 1.0502 & 1.13703 \\
\hline & & MSE & 0.051584 & 0.13396 & 0.021163 & 0.021163 & 0.015552 & 0.10892 & 0.025639 & 0.025639 & 0.016705 \\
\hline \multirow{4}{*}{$\begin{array}{l}(30,40) \\
(20,30) \\
\end{array}$} & \multirow{2}{*}{$\begin{array}{c}2 \\
(15,20)\end{array}$} & & 112 & & 1.16 & 1.16014 & & 1.66 & 1.14725 & 1.14725 & 1.20395 \\
\hline & & MSE & 0.044112 & 0.24434 & 0.003043 & 0.003043 & 0.001933 & 0.21826 & 0.004199 & 0.004199 & 0.001575 \\
\hline & \multirow{2}{*}{2.5} & Mean & 0.949765 & 1.51148 & 1.03687 & 1.03687 & 1.08872 & 1.4904 & 1.02689 & 1.02689 & 1.07772 \\
\hline & & MSE & 0.06293 & 0.10428 & 0.030028 & 0.030028 & 0.016152 & 0.09108 & 0.033216 & 0.033216 & 0.018525 \\
\hline \multirow{4}{*}{$\begin{array}{r}(40,50) \\
(30,40) \\
\end{array}$} & \multirow{2}{*}{$\begin{array}{c}2 \\
(20,30)\end{array}$} & Mean & 1.08732 & 1.55271 & 1.06516 & 1.06516 & 1.11842 & 1.53422 & 1.0564 & 1.0564 & 1.10877 \\
\hline & & MSE & 0.045626 & 0.13346 & 0.022442 & 0.022442 & 0.011353 & 0.12031 & 0.024734 & 0.024734 & 0.012851 \\
\hline & \multirow{2}{*}{2.5} & Mean & 1.03461 & 1.4691 & 1.0078 & 1.0078 & 1.05819 & 1.45211 & 0.999774 & 0.999774 & 1.04935 \\
\hline & & MSE & 0.038813 & 0.07508 & 0.038195 & 0.038195 & 0.021492 & 0.06612 & 0.041312 & 0.041312 & 0.024042 \\
\hline
\end{tabular}

TABLE 3: Real data set: average estimates, MSE of MLEs, Bayesian, and E-Bayesian estimates for $\theta$ under LINEX and SEL functions when $\theta=2, \lambda=1.24, a_{1}=0.8, b_{1}=0.7, a_{2}=1.6, b_{2}=1.4, h=1.5$, and $c_{1}=c_{2}=2$.

\begin{tabular}{|c|c|c|c|c|c|c|c|c|c|c|c|}
\hline \multirow{2}{*}{$\begin{array}{l}\left(n_{1}, n_{2}\right) \\
\left(r_{1}, r_{2}\right)\end{array}$} & \multirow{2}{*}{$\begin{array}{c}T \\
\left(k_{1}, k_{2}\right)\end{array}$} & \multirow{2}{*}{ Criteria } & \multirow{2}{*}{$\widehat{\theta}_{M L E}$} & \multicolumn{4}{|c|}{ Squared error loss } & \multicolumn{4}{|c|}{ LINEX loss } \\
\hline & & & & $\widehat{\theta}_{B S}$ & $\widehat{\theta}_{E B S 1}$ & $\widehat{\theta}_{E B S 2}$ & $\widehat{\theta}_{E B S 3}$ & $\widehat{\theta}_{B L}$ & $\widehat{\theta}_{E B L 1}$ & $\widehat{\theta}_{E B L 2}$ & $\widehat{\theta}_{E B L 3}$ \\
\hline$(26,30)$ & 4.8 & Mean & 2.5674 & 2.67282 & 1.83355 & 1.83355 & 1.92523 & 2.62482 & 1.81165 & 1.81165 & 1.901 \\
\hline$(21,25)$ & $(15,20)$ & MSE & 0.321945 & 0.45272 & 0.027722 & 0.027722 & 0.005609 & 0.39045 & 0.035495 & 0.035495 & 0.009824 \\
\hline$(26,30)$ & 5.8 & Mean & 2.57821 & 2.67914 & 1.83789 & 1.83789 & 1.92978 & 2.6324 & 1.81658 & 1.81658 & 1.90621 \\
\hline$(21,25)$ & $(15,20)$ & MSE & 0.334329 & 0.46127 & 0.026299 & 0.026299 & 0.004951 & 0.39998 & 0.033665 & 0.033665 & 0.008821 \\
\hline
\end{tabular}

TABLE 4: Real data set: average estimates, MSE of MLEs, Bayesian, and E-Bayesian estimates for $\lambda$ under LINEX and SEL functions when $\theta=2, \lambda=1.24, a_{1}=0.8, b_{1}=0.7, a_{2}=1.6, b_{2}=1.4, h=1.5$, and $c_{1}=c_{2}=2$.

\begin{tabular}{cccccccccccc}
\hline$\left(n_{1}, n_{2}\right)$ & $T$ & \multirow{2}{*}{ Criteria } & $\hat{\lambda}_{M L E}$ & $\hat{\lambda}_{B S}$ & $\hat{\lambda}_{E B S 1}$ & $\hat{\lambda}_{E B S 2}$ & $\hat{\lambda}_{E B S 3}$ & $\hat{\lambda}_{B L}$ & \multicolumn{2}{c}{ LINEX loss } \\
$\left(r_{1}, r_{2}\right)$ & $\left(k_{1}, k_{2}\right)$ & & & & & $\hat{\lambda}_{E B L 1}$ & $\hat{\lambda}_{E B L 2}$ & $\hat{\lambda}_{E B L 3}$ \\
\hline$(26,30)$ & 4.8 & Mean & 1.02772 & 1.06214 & 1.08763 & 1.08763 & 1.30515 & 1.05994 & 1.08533 & 1.08533 & 1.30187 \\
$(21,25)$ & $(15,20)$ & MSE & 0.045063 & 0.03164 & 0.023226 & 0.023226 & 0.023226 & 0.004258 & 0.023932 & 0.023932 & 0.00384 \\
$(26,30)$ & 5.8 & Mean & 1.02965 & 1.06248 & 1.08798 & 1.08798 & 1.30558 & 1.06027 & 1.08566 & 1.08566 & 1.30227 \\
$(21,25)$ & $(15,20)$ & MSE & 0.044246 & 0.03152 & 0.02312 & 0.02312 & 0.004316 & 0.03231 & 0.023829 & 0.023829 & 0.003891 \\
\hline
\end{tabular}


from the State of So Paulo, including a period of 56 years from 1947 to 2003. Also, they checked the fitting of the given data set through different method of estimation and stated that EGD gives a good fit for these data. These data contain 56 observations listed as follows: 0.2, 3.5, 2.8, 3.7, 8.7, 6.9, 7.4, $0.8,4.8,2.5,2.9,3.1,4.0,5.0,3.8,3.5,5.4,3.3,2.9$, $1.7,7.3,2.9,4.6,1.1,1.4,3.9,6.2,4.1,10.8,3.8,7.3,1.8,6.7,3.5$, $3.2,5.2,2.8,5.2,5.4,2.2,9.9,2.1,4.7,5.5,2.6,4.1,5.4,5.5,2.1$, $1.9,8.8,1.3,24.1,5.4,6.2,2.9$.

We suppose that values of data set represent lifetime of failure observations which follow the EGD. Now we will apply the CSPALT when the sample is genralized Type-I hybrid censoring scheme. Such that $n_{1}=26$ and $n_{2}=30$, where the first sample and the second sample were selected randomly from the complete sample of size $n=56$ units. We desire to obtain $r_{1}=21$ of failures out of $n_{1}=26$ units, and $k_{1}=15$ is a bare minimum number of failures that can be accepted out of $n_{1}=26$ units. While we desire to obtain $r_{2}=$ 25 failures out of $n_{2}=30$ units, $k_{2}=20$ represents a minimum number of failures is acceptable from $n_{2}=30$ units. All estimates of $\theta$ and $\lambda$ are derived based on the same previous procedures and shown in Tables 3 and 4. Table 3 gives estimates and MSE for the parameter $\theta$ of ML, Bayesian, and E-Bayesian estimation methods based on SEL and LINEX loss functions. Table 4 gives the previous criteria for the acceleration factor $\lambda$. By observing results listed in Tables 3 and 4 regarding the real data set, one can note that the E-Bayesian method is the best compared with both ML and Bayesian estimation methods because of having less MSE. Moreover, the proposed methods are easily applied to the real data and gave good results.

\section{Conclusion}

In this paper, we studied the exponentiated gamma distribution (EGD) with generalized Type-I hybrid censored data under the constant-stress partially accelerated life test (CSPALT) model. We discussed the Bayesian and E-Bayesian estimation methods, as well as the maximum likelihood method, for the distribution parameter and the acceleration factor. The E-Bayesian and Bayesian estimates are obtained by the SEL and the LINEX loss functions. The MCMC method is used for deriving the Bayesian estimates, and then we computed the E-Bayesian estimates. We provided a real data set to clarify the behavior of the methods in the application.

From the results shown in Tables 1-4, we may conclude that the E-Bayesian estimation approach is superior to both ML and Bayesian estimation methods due to its lower MSE. Also, the E-Bayesian estimation method is easy to be applied and convenient to the application. Additionally, by including additional failure items in the CSPALT model with censoring strategies, adequate information about test units is obtained. Additionally, it is shown that the presented methodologies are simply applicable to the CSPALT model and provide acceptable results.

\section{Data Availability}

The data used to support this study are included within the article.

\section{Conflicts of Interest}

The authors declare that they have no conflicts of interest regarding the publication of this research.

\section{Acknowledgments}

This study was funded by Taif University Researchers Supporting Project number TURSP-2020/279, Taif University, Taif, Saudi Arabia.

\section{References}

[1] B. Epstein, "Truncated life tests in the exponential case," The Annals of Mathematical Statistics, vol. 25, no. 3, pp. 555-564, 1954.

[2] B. Chandrasekar, A. Childs, and N. Balakrishnan, "Exact likelihood inference for the exponential distribution under generalized Type-I and Type-II hybrid censoring," Naval Research Logistics, vol. 51, no. 7, pp. 994-1004, 2004.

[3] W. T. Huang and K. C. Yang, "A new hybrid censoring scheme and some of its properties," Tamsui Oxford Journal of Mathematical Sciences, vol. 26, no. 4, pp. 355-367, 2010.

[4] A. Rabie and J. Li, "E-bayesian estimation based on burr-X generalized type-II hybrid censored data," Symmetry, vol. 11, pp. 1-14, 2019.

[5] A. Rabie and J. Li, "E-bayesian estimation for burr-X distribution based on generalized type-I hybrid censoring scheme," American Journal of Mathematical and Management Sciences, vol. 39, no. 1, pp. 41-55, 2020.

[6] W. Nelson, Accelerated Life Testing: Statistical Models, Data Analysis and Test Plans, John Wiley and Sons, New York, NY, USA, 1990.

[7] R. C. Gupta, P. L. Gupta, and R. D. Gupta, "Modeling failure time data by Lehman alternatives," Communications in Statistics - Theory and Methods, vol. 27, no. 4, pp. 887-904, 1998.

[8] A. I. Shawky and R. A. Bakoban, "Bayesian and non-bayesian estimations on the exponentiated gamma distribution," Applied Mathematical Sciences, vol. 2, no. 51, pp. 2521-2530, 2008.

[9] I. Shawky and R. A. Bakoban, "Order statistics from exponentiated gamma distribution and associated inference," International Journal of Contemporary Mathematical Sciences, vol. 4, no. 2, pp. 71-91, 2009.

[10] I. Shawky and R. A. Bakoban, "On finite mixture of twocomponent exponentiated gamma distribution," Journal of Applied Sciences Research, vol. 5, no. 10, pp. 1351-1369, 2009.

[11] S. K. Singh, U. Singh, and D. Kumar, "Bayesian estimation of the exponentiated gamma parameter and reliability function under asymmetric loss function," REVSTAT-Statistical Journal, vol. 9, no. 3, pp. 247-260, 2011.

[12] R. U. Khan and D. Kumar, "Lower generalized order statistics from exponentiated gamma distribution and its characterization. ProbStat forum," vol. 4, pp. 25-38, 2011.

[13] H. P. Ghanizadeh and R. Lotfi, "Classical estimations of the exponentiated gamma distribution parameters with presence of K outliers," Australian Journal of Basic and Applied Sciences, vol. 5, no. 3, pp. 571-579, 2011.

[14] N. Feroze and M. Aslam, "Bayesian analysis of exponentiated gamma distribution under type II censored samples," Scientific Journal of Pure and Applied Sciences, vol. 1, no. 1, pp. 30-39, 2012. 
[15] M. Han, "The structure of hierarchical prior distribution and its applications," Chinese Operation Research and Management Science, vol. 6, pp. 31-40, 1997.

[16] M. Han, "E-Bayesian estimation and hierarchical Bayesian estimation of failure rate," Applied Mathematical Modelling, vol. 33, no. 4, pp. 1915-1922, 2009.

[17] Z. F. Jaheen and H. M. Okasha, "E-Bayesian estimation for the Burr type XII model based on type-2 censoring," Applied Mathematical Modelling, vol. 35, no. 10, pp. 4730-4737, 2011.

[18] H. M. Okasha, "E-Bayesian estimation for the Lomax distribution based on type-II censored data," Journal of the Egyptian Mathematical Society, vol. 22, no. 3, pp. 489-495, 2014.

[19] A. Rabie and J. Li, "E-Bayesian estimation for Burr-X distribution based on Type-I hybrid censoring scheme," IAENG International Journal of Applied Mathematics, vol. 48, Article ID 244250, 2018.

[20] A. Rabie and J. Li, "Inferences for burr-X model based on unified hybrid censored data," IAENG International Journal of Applied Mathematics, vol. 49, pp. 561-567, 2019.

[21] A. Rabie, "E-Bayesian estimation for a constant-stress partially accelerated life test based on Burr-X Type-I hybrid censored data," Journal of Statistics and Management Systems, vol. 24, no. 8, pp. 1649-1667, 2021.

[22] S. K. Singh, U. Singh, and A. S. Yadav, "Bayesian estimation for exponentiated gamma distribution under progressive type-II censoring using different approximation techniques," Journal of Data Science, vol. 13, pp. 551-568, 2015. 